\title{
Response readiness modulates the development of association-based automaticity in masked priming
}

\author{
Yongchun Wang ${ }^{1,2}$ - Yonghui Wang ${ }^{1,2} \cdot$ Peng Liu $^{3}$. \\ Meilin Di ${ }^{1,2}$ - Yanyan Gong ${ }^{1,2} \cdot$ Li Zhao $^{1,2} \cdot$ Qi Chen $^{1,2}$
}

Published online: 11 January 2017

(C) The Psychonomic Society, Inc. 2017

\begin{abstract}
The current study investigated the role of the automatization of stimulus and response (S-R) associations and response readiness in triggering the motor activation for masked primes in two experiments. The automatization of associations was manipulated by employing different types of stimuli, and response readiness was manipulated by varying the relative frequency of Go trials in a modified Go/No-Go task. Compatibility (compatible and incompatible), stimulus type (arrows and parallel lines), and test session (Sessions 1, 2, and 3) were manipulated in a high response-readiness condition (Experiment 1) and in a low response-readiness condition (Experiment 2). Negative compatibility effects (NCEs) occurred regardless of session and experiment in the arrow stimuli condition. However, in the parallel-line stimuli condition, no significant compatibility effect (CE) appeared regardless of the experiment in Sessions 1 and 2, whereas a significant NCE appeared in Experiment 1 but not in Experiment 2 in Session 3 . These results are consistent with the claim that motor activation can only occur if the association between specific stimuli and specific responses has been automatized by previous practice, and response readiness can modulate the development of automaticity, but this modulation will have a minimal effect once the association is automatized. The findings also
\end{abstract}

Yonghui Wang

wyonghui@snnu.edu.cn

1 School of Psychology, Shaanxi Normal University, Xi'an 710062, China

2 Shaanxi Provincial Key Laboratory of Behavior and Cognitive Neuroscience, Xi'an 710062, China

3 School of Public Management, Northwest University, Xi'an 710069, China provide experimental evidence for the assumption that the formation of association-based automaticity could be modulated by top-down control (e.g., response readiness).

Keywords Motor activation - Automatization of associations $\cdot$ Response readiness $\cdot$ Negative compatibility effect

\section{Introduction}

The negative compatibility effect (NCE), which was first reported by Eimer and Schlaghecken (1998), refers to the phenomenon in which responses to the target are faster (and more accurate) when a target is preceded by an incompatible prime (i.e., cueing an opposite response to the target) and slower (and less accurate) when a target is preceded by a compatible prime (i.e., cueing the same response as the target). A typical NCE paradigm is described as follows. First, a prime, such as a double-headed arrow that points left or right, is presented in the center of a screen for a brief time (typically less than $35 \mathrm{~ms}$ ). Second, a mask, such as a stimulus constructed of randomly orientated lines, is presented at the prime's location for approximately $100 \mathrm{~ms}$. Finally, a target is presented for $100 \mathrm{~ms}$. Participants are asked to respond to the target's direction as quickly and accurately as possible. The compatibility effect (CE) is measured by evaluating the response time (RT) to the target between the compatible and incompatible trials. It should be noted that the NCE is absent at very short primetarget stimulus onset asynchronies (SOAs; $60 \mathrm{~ms}$ or less); instead, the positive compatibility effect (PCE, which indicates that viewers exhibit shorter RTs when responding to targets when these targets are preceded by compatible primes and exhibit delayed responses if the targets are preceded by incompatible primes) is observed. 
To account for their results, Eimer and Schlaghecken (1998) proposed a self-inhibition (SI) hypothesis of the NCE. They considered that motor activation by a subliminal prime is automatic and produces an inhibition that counteracts the initial activation, provided that perceptual evidence for the prime is sufficiently strong and is immediately removed (Bowman, Schlaghecken, \& Eimer, 2006; Eimer, 1999; Eimer \& Schlaghecken, 1998, 2002; Klapp, 2005; Klapp \& Hinkley, 2002; Schlaghecken \& Eimer 2002, 2004, 2006). It is an activation-followed-by-inhibition process. If a compatible target is presented during the inhibitory phase, the required response remains inhibited, which produces the NCE. The self-inhibition is triggered by motor activation of the prime; thus, the strength of inhibition is intimately related to the initial motor activation. Specifically, strong motor activations trigger strong inhibitions when the supporting sensory evidence is suddenly removed (i.e., when primes are successfully backward-masked) and cause increased NCEs. Conversely, weak motor activations trigger weak inhibitions. However, when the motor activation is too weak, it will not trigger inhibition because activation cannot cross the inhibition threshold (Schlaghecken \& Eimer, 2000, 2002).

An alternative viewpoint of the NCE inhibitory mechanism was suggested by Jaśkowski and colleagues (Jaśkowski, 2007, 2008, 2009; Jaśkowski, Białuńska, Tomanek, \& Verleger, 2008; Jaśkowski \& Slósarek, 2007; Jaśkowski \& Verleger, 2007), who proposed the mask-triggered inhibition (MTI) hypothesis. Similar to the SI hypothesis, this hypothesis postulates that an initial activation is replaced by inhibition. However, the MTI hypothesis assumes that the stimulus that appears between the prime and the target often masks the prime stimulus, but this masking function is not the cause of sign reversal. Instead, an intervening stimulus triggers inhibition independent of masking (Klapp, 2015; see also Panis \& Schmidt, 2016; Schmidt, Hauch, \& Schmidt, 2015). Jaśkowski (2007) stated "an intervening stimulus can modulate priming even if this stimulus does not mask the prime and even if it contains no features relevant to the participants' task" (i.e., irrelevant mask). He also noted that masktriggered inhibition may be stronger with relevant masks than with irrelevant masks.

Substantial research has demonstrated that an NCE only occurs if the associations between the stimuli that are used as primes and the responses (S-R) have been automatized by previous responses to these stimuli (Boy \& Sumner, 2010; Klapp, 2015; Schlaghecken, Blagrove, \& Maylor, 2007; Schlaghecken \& Eimer, 2002). Boy and Sumner (2010) stated "When associations between certain visual stimuli and particular actions are learned, those stimuli become capable of automatically and unconsciously activating their associated action plans," the NCE will be observed. Schlaghecken and Eimer (2002) have confirmed that once the association becomes sufficiently strong to exceed the inhibitory threshold, self-inhibition is produced, and the NCE will occur. In addition, Klapp (2015) has proposed self-automatization in which the associations produced by responding to target stimuli of the same type as the stimuli used in primes become automatized when the same set of stimuli are used for both primes and targets. Therefore, in this case, automatization can be produced by responding in these single trials. When different sets of stimuli are used for primes and targets, automatization can also be produced by responding in intermixed auxiliary trials. For example, in Klapp and Greenberg (2009), the task only contains primes and targets. Letter-arrow trials (which were not self-automatized) were accompanied by randomly intermixed auxiliary trials (the prime was denoted by $*_{* *}$, and the target was denoted by two letters) that required responses to letter targets. These auxiliary trials automated the associations between letters and their corresponding responses to ensure that substantial PCE was produced in the subsequent letter-arrow test trial. Moreover, some studies have confirmed that the associative strength of the stimuli that naturally contain consistent directions with responses (left or right), e.g., arrows, is stronger than that of the stimuli that do not contain consistent directions with responses, e.g., letter, line, or digit stimuli. And practice is a useful way to strengthen the weak associations, which results in a robust and persistent effect (Boy \& Sumner, 2010; Klapp, 2005, 2015; Klapp \& Haas, 2005; Klapp \& Hinkley, 2002; Liu \& Wang, 2014; Neill, Terry, \& Valdes, 1994; Neumann, 1984; Sumner, 2008; Tipper et al., 1991). For example, Klapp (2015) stated "Practice using the association between a stimulus, the prime, and a response is needed to produce automatic activation." Boy and Sumner (2010) found that the weaker associations of lines are reinforced over several blocks.

Response readiness is another factor that may facilitate the triggering of motor activations. Response readiness has generally been identified as an anticipatory pre-activation of response structures that are induced and modified not only by internal factors such as intention or motivation but also by external factors such as instruction or task demands (i.e., top-down). However, such structures are not affected by stimulus types (Miller, 1983; Schlaghecken \& Eimer, 2001). Some studies have suggested that weak stimuli can trigger their corresponding motor activations if they accompany a state of high response readiness (Brunia, 1997; Coles, Gratton, Bashore, Eriksen, \& Donchin, 1985; Dosher \& Lu, 2000; Gratton, Coles, Sirevaag, Eriksen, \& Donchin, 1988; Rosenbaum, 1980; Welsh \& Elliott, 2004). For example, Coles, Gratton, Bashore, Eriksen, and Donchin (1985) used letter stimuli (HHHHH, SSHSS, SSSSS, and HHSHH) and reported that motor responses required less stimulus-related activation for their evocation in a state of high response readiness (i.e., in the blocks in which a warning tone preceded the stimulus arrays) than in a state of low response readiness (i.e., in the blocks in which no warning tone was given). 
Additionally, Gratton, Coles, Sirevaag, Eriksen, and Donchin (1988) used the same letter stimuli and deduced that response readiness is an energizing phenomenon through which response structures are activated at a sub-threshold level. Therefore, these studies assumed that stimuli that are perceptually extremely weak, such as successfully masked primes, could trigger their corresponding motor activations only if the motor system is already in a state of high response readiness.

Unfortunately, the automatization of associations could be variable and may influence the motor activations in the previously mentioned studies. For example, in Coles, Gratton, Bashore, Eriksen, and Donchin (1985), the associations between letter stimuli and responses can be automatized by responding to the stimuli. The associations should be readily automatized in the state of high response readiness (i.e., at a high level of alertness, in which a warning tone preceded the stimulus arrays) because alertness may facilitate learning to quickly and effectively provide specific responses to specific stimuli (Buckner \& McGrath, 1963; Posner, 1980; Posner \& Petersen, 1990). Another possibility is that high response readiness might lower the response threshold. Thus response readiness may not directly influence motor activations.

This speculation is also corroborated by other research findings that confirm that response readiness does not affect the initial motor activation when the associations are automatized (Eimer \& Schlaghecken 1998; Miller, 1983; Schlaghecken \& Eimer, 2001). For example, Schlaghecken and Eimer (2001) used arrow stimuli (whose association is highly compatible) in the NCE paradigm and manipulated the overall response readiness by varying the probability of Go trials in a choice reaction Go/No-Go task. The results showed that the NCEs were not affected by the state of response readiness, although the mean RTs were longer for the state of low response readiness than for the state of high response readiness. This finding indicated that motor activation is independent of response readiness when the associations are stronger and also suggested that the state of response readiness could not affect the response threshold because it should affect the NCE regardless of stimulus type (e.g., letter or arrow stimuli) if it affects the response threshold.

Based on the researches and summaries above, response readiness could not influence motor activation when the associations are stronger, whereas it may indirectly influence motor activation when they are weaker, because the associative strength for arrows is stronger than that for letter stimuli. Accordingly, the current study focused on the role of the automatization of associations and response readiness in triggering the motor activation for masked primes. The relative frequency of Go trials in a modified Go/No-Go task was varied as in Schlaghecken and Eimer (2001), with two methodological changes. First, the stimuli that naturally contain consistent directions with responses, i.e., arrows, and the stimuli that do not contain consistent directions with responses, i.e., vertical and horizontal parallel lines, were used to distinguish the different strengths of the associations. Second, to slowly establish the associations between the stimuli of vertical and horizontal parallel lines and their corresponding responses, the experiments were conducted over three successive sessions with the goal of providing extensive practice (see Boy \& Sumner, 2010). Response readiness was manipulated by varying the probability of the Go trials in a choice reaction Go/NoGo task. Response readiness was assumed to be high when the probability of Go trials was high (80\% in Experiment 1), whereas a low probability of Go trials (20\% in Experiment 2) should reduce response readiness (see Low \& Miller, 1999; Schlaghecken \& Eimer, 2001). Note that the two experiments involved an equal number of Go trials. Parallel lines or arrows were used as stimuli. The second variable was the congruency of the prime and target. In the two experiments, the compatible and incompatible trials occurred randomly, with equal probability within each block. The third variable was a test session (three successive test sessions of Sessions 1, 2, and 3).

If the automatization of associations played a causal role in triggering motor activations, we predicted that similar patterns of results would be observed between Experiment 1 (Go 80\%) and Experiment 2 (Go 20\%) because these two experiments featured an equal number of Go trials. In the parallel-line stimuli condition, we predicted that no significant NCE would appear regardless of the experiment (Go $80 \%$ or Go $20 \%$ ) early in practice (e.g., Session 1) because the S-R associations should be too weak to trigger an inhibition process in this scenario. Furthermore, we predicted that the NCE would appear regardless of experiment (Go $80 \%$ or Go $20 \%$ ) in subsequent sessions (especially Session 3) because the strength of the S-R associations should increase through practice, and the motor activation for the prime should exceed the inhibitory threshold. In the arrow stimuli condition, however, we predicted that significant NCEs would emerge regardless of session stage (early or subsequent sessions) and experiment (Go $80 \%$ or Go $20 \%$ ); moreover, there should be no significant difference among experiments and sessions. These outcomes are predicted because the strength of the associations should be sufficiently strong for the traditional arrow stimuli, which naturally contain consistent directions with the responses.

If response readiness was the only factor that played a causal role in triggering motor activations, we predicted that similar patterns of results would be observed between the parallel-line stimuli condition and the arrow stimuli condition because response readiness is not affected by stimulus type. In Experiment 1 (Go 80\%), we predicted that the NCE would appear regardless of stimulus type (lines or arrows) and session stage (early or later sessions) because response readiness should be high in the Go $80 \%$ experiment. However, in Experiment 2 (Go 20\%), we predicted that no significant NCE would appear regardless of stimulus type (lines or 
arrows) and session stage (early or later sessions) because response readiness should be low in the Go $20 \%$ experiment.

To yield an equal number of Go trials between Experiments 1 and 2, the number of No-Go trials in Experiment 2 must be four times that in Experiment 1. Schlaghecken et al. (2008) indicated that perceptual learning occurs in a prolonged standard forced-choice task (FC task, which is designed to assess prime visibility, i.e., masking effectiveness) if the masking stimulus remains constant across trials. Perceptual learning has been identified as the "increase in the ability to extract information from the environment as a result of practice and experience with stimulation coming from it" (Gibson, 1969). This type of learning enables participants to eventually correctly identify and subjectively perceive a masked stimulus despite being unaware of it and unable to respond with better-than-chance accuracy at the beginning of the experiment (Schlaghecken et al., 2008). In the current study, this raises the question of whether No-Go trials can lead to perceptual learning, as observed in FC trials. If perceptual learning also occurred in the present study, its strength would be greater in Experiment 2 than in Experiment 1, and prime visibility would be higher in Experiment 2 than in Experiment 1. Thus, perceptual learning may be an uncontrolled variable that could bias our results in distinguishing between the automatization of associations and response readiness. To address this issue, a standard FC task followed the primary task in both Experiments 1 and 2. The FC task had the following two purposes: first, to assess prime visibility, i.e., masking effectiveness; and second, to investigate whether the strength of perceptual learning is stronger in Experiment 2 than in Experiment 1.

\section{Experiment 1}

\section{Methods}

\section{Participants}

Twenty-four paid college students (nine male) who ranged in age from 18 to 30 years (mean age $=20.79$ years) participated in the experiment. All of the participants were right-handed and had normal or corrected-to-normal vision. Informed consent was obtained from each participant at the start of the experimental session.

\section{Stimuli and apparatus}

In the parallel-line stimuli condition, vertical and horizontal parallel lines (= and $\|)$ were used as the primes. The masks were randomly filled with 42 oblique lines (i.e., excluding any orientation closer than $\pm 5^{\circ}$ to the vertical or the horizontal lines) with six different lengths that ranged from $0.1^{\circ}$ to $0.6^{\circ}$. Vertical parallel, horizontal parallel, and mutually perpendicular lines $(=, \|$, and +$)$ served as the targets. In the arrow stimuli condition, left- and right-pointing double arrows $(<<$ and $\gg>$ ) were used as the primes. The masks were randomly filled with 42 vertical and horizontal lines with six different lengths that ranged from $0.1^{\circ}$ to $0.6^{\circ} \cdot{ }^{11}$ Left-pointing, rightpointing, and inward-pointing double arrows $(<<,>>$, and $><)$ served as the targets. The primes and targets subtended a visual angle of approximately $1^{\circ} \times 0.35^{\circ}$, and the masks subtended a visual angle of $2.2^{\circ} \times 2.2^{\circ}$. All stimuli were presented in white on a black background. The experiment was performed on a PC that run at $60 \mathrm{~Hz}$ and was linked to a 17 -in. CRT monitor.

\section{Procedure}

The participants were seated in a dimly lit chamber and placed in front of a monitor, with a $60-\mathrm{cm}$ distance between the screen and their eyes; the center of the screen was located at the center of their horizontal sightline at a fixed, straight-ahead level. The experiment was divided into two tasks, namely, a primary task (the NCE task) and an FC task (a test of prime visibility).

The trial procedure of the primary task is shown in Fig. 1. Each trial began with a central fixation cross $(300 \mathrm{~ms})$, and then a prime was presented for $17 \mathrm{~ms}$, followed by a mask $(100 \mathrm{~ms})$. The mask appeared at the same spatial location as the prime to ensure that the prime was occluded. Finally, a target stimulus was presented for $100 \mathrm{~ms}$. The participants were instructed to maintain central eye fixation and to respond as quickly and accurately as possible to the left-pointing or right-pointing double arrows or the vertically parallel or horizontally parallel line targets (Go stimuli) that were presented in each trial but not to respond to the inward-pointing arrows or mutually perpendicular line targets (No-Go stimuli). The No-Go targets were presented in $20 \%$ of all trials, and the Go targets were presented in $80 \%$ of all trials. By using an English keyboard, the participants were instructed to use the index finger of their left hand to respond with a $\mathrm{Z}$ key-press to " $<<"$ or "=" and to use the index finger of their right hand to respond with an M key-press to " $>>$ " or "||". At the beginning of experiment, the participants were informed that $80 \%$ of the trials were Go trials.

In the primary task, parallel-line and arrow stimuli were used. These two stimuli conditions divided one session into

\footnotetext{
${ }^{1}$ The choice of different masks (i.e., irrelevant masks) in the two stimulus conditions excludes the effects that are induced by object updating $(\mathrm{OU})$. The OU account assumed that once the masks share features with the primes (e.g., arrow primes and oblique-lines masks, or vertical and horizontal-lines primes and masks), OU will occur. The updating features, including features that indicate a direction opposite to the direction of the prime, should improve a participant's response to a target for which the direction is opposite the direction of the prime, thus causing the NCE.
} 


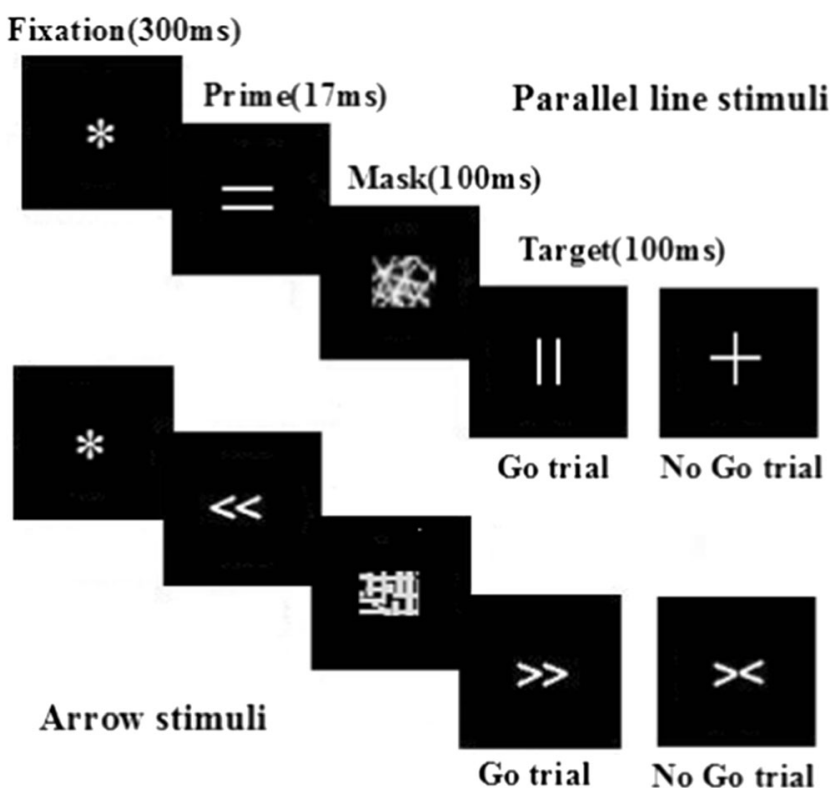

Fig. 1 Schematic representation of the trial procedure in the Experiments

two blocks (60 trials each). Compatibility was randomized and equiprobable within each block. The sequence of parallel-line and arrow stimuli blocks was completely randomized for each participant. The inter-trial-interval (ITI) was 1,500 ms. A 16-trial practice phase was presented before the formal experiment, and the formal experiment did not begin unless the correct rate in practice exceeded $90 \%$. The participants were given a rest period of at least 2 min after each block and encouraged to take longer breaks whenever necessary. The tasks were completed in three successive sessions for each participant to strengthen the $\mathrm{S}-\mathrm{R}$ associations through practice. The participants were given a rest period of at least 3 min after each session.

The secondary task was an FC task. It was scheduled to occur following the primary task in the third session of the experiment. Two blocks (30 trials each), a parallel-line block and an arrow block, were assigned; the block order was counter balanced. The trial arrangement was similar to the primary task except that no target was presented. The participants were instructed to react to the primes as they did to the targets in the primary task. They were told to execute each response after the mask presentation and encouraged to guess if they could not clearly identify the prime.

\section{Results}

The performance of the FC task was assessed as follows: arrow stimuli condition, $50.42 \%$, and parallel-line stimuli condition, $48.47 \%$. Neither of the two conditions enabled the primes to significantly differ from chance $(50 \%)$, all were $t$ s $(23)<1.66$ and $p s>.11$, which suggests that all primes were processed without awareness.
In the primary task, the RTs of correct responses, the arcsine-transformed error rates and Miss rates on Go trials and the arcsine-transformed False Alarm rates on the No-Go trials were analyzed. Furthermore, we excluded outside of plus and minus three standard deviations from the RTs in each session for each participant (in total, $0.85 \%$ of the GO trials). Repeated measure analyses of variance (ANOVAs) were computed for the RTs of correct responses, arcsine-transformed error rates and Miss rates on Go trials for the factors of test session (Sessions 1, 2, and 3), stimulus type (arrows, parallel lines) and compatibility (compatible, incompatible). A Repeated measure ANOVA was computed for the arcsinetransformed False Alarm rates on No-Go trials for the factors of test session (Sessions 1, 2, and 3) and stimulus type (arrows, parallel lines). Concerning the RT, a significant main effect of compatibility $[\mathrm{F}(1,23)=7.15, p=.014$, MSE $=$ $\left.476.51, \eta_{\mathrm{p}}{ }^{2}=.24\right]$ was observed, showing that the mean RT for incompatible trials (mean $=502 \mathrm{~ms}$ ) was shorter than that for compatible trials (mean $=509 \mathrm{~ms}$ ). No significant difference was observed between stimulus types $[\mathrm{F}(1,23)=1.19, p$ $=.29, \mathrm{MSE}=1840.51]$ or among the three sessions $[\mathrm{F}(1.84$, $42.39)^{\mathrm{g}}=.36, p=.68, \mathrm{MSE}=9813.08$ ] (the " $\mathrm{g}$ " superscript indicates the use of Greenhouse-Geisser adjustments to the degrees of freedom). More importantly, the analysis revealed a significant three-way interaction $\left[\mathrm{F}(1.94,44.66)^{\mathrm{g}}=4.29, p\right.$ $\left.=.02, \mathrm{MSE}=312.35, \eta_{\mathrm{p}}{ }^{2}=.16\right]$. Furthermore, the two-way interaction between stimulus type and compatibility was significant $\left[\mathrm{F}(1,23)=13.46, p=.001, \mathrm{MSE}=202.09, \eta_{\mathrm{p}}{ }^{2}=\right.$ $.37]$, and the two-way interaction between test session and compatibility was marginally significant $\left[\mathrm{F}(1.92,44.08)^{\mathrm{g}}=\right.$ $\left.3.05, p=.06, \mathrm{MSE}=520.94, \eta_{\mathrm{p}}{ }^{2}=.12\right]$. The two-way interaction between test session and stimulus type was not significant $\left[\mathrm{F}(1.39,31.98)^{\mathrm{g}}=.15, p=.79, \mathrm{MSE}=4937.48\right]$. Next, we examined the interaction between stimulus type and compatibility by session. Figure 2 details this relationship across the three sessions. In Session 1, the two-way interaction between stimulus type and compatibility was significant [F (1, $\left.23)=12.15, p=.002, \mathrm{MSE}=227.76, \eta_{\mathrm{p}}{ }^{2}=.35\right]$. Subsequent paired $t$-tests confirmed a significant NCE in the arrow condition $[t(23)=2.41, p=.024, \mathrm{SE}=5.29]$, showing that the mean RT for incompatible trials (mean $=500 \mathrm{~ms}$ ) was shorter than that for compatible trials (mean $=513 \mathrm{~ms}$ ), but no significant $\mathrm{CE}$ in the line condition $[t(23)=1.82, p=.081, \mathrm{SE}=$ 4.78]. In Session 2, the two-way interaction between stimulus type and compatibility was significant $[\mathrm{F}(1,23)=9.99, p=$ $\left..004, \mathrm{MSE}=242.56, \eta_{\mathrm{p}}{ }^{2}=.30\right]$. Subsequent paired $t$-tests confirmed a significant NCE in the arrow condition $[t(23)=$ 2.60, $p=.016, \mathrm{SE}=4.85]$, showing that the mean RT for incompatible trials (mean $=492 \mathrm{~ms}$ ) was shorter than that for compatible trials $($ mean $=505 \mathrm{~ms}$ ), but no significant $\mathrm{CE}$ in the line condition $[t(23)=1.41, p=.17, \mathrm{SE}=5.32]$. In Session 3, the two-way interaction between stimulus type and compatibility was not significant $[\mathrm{F}(1,23)=.39, p=.54$, 

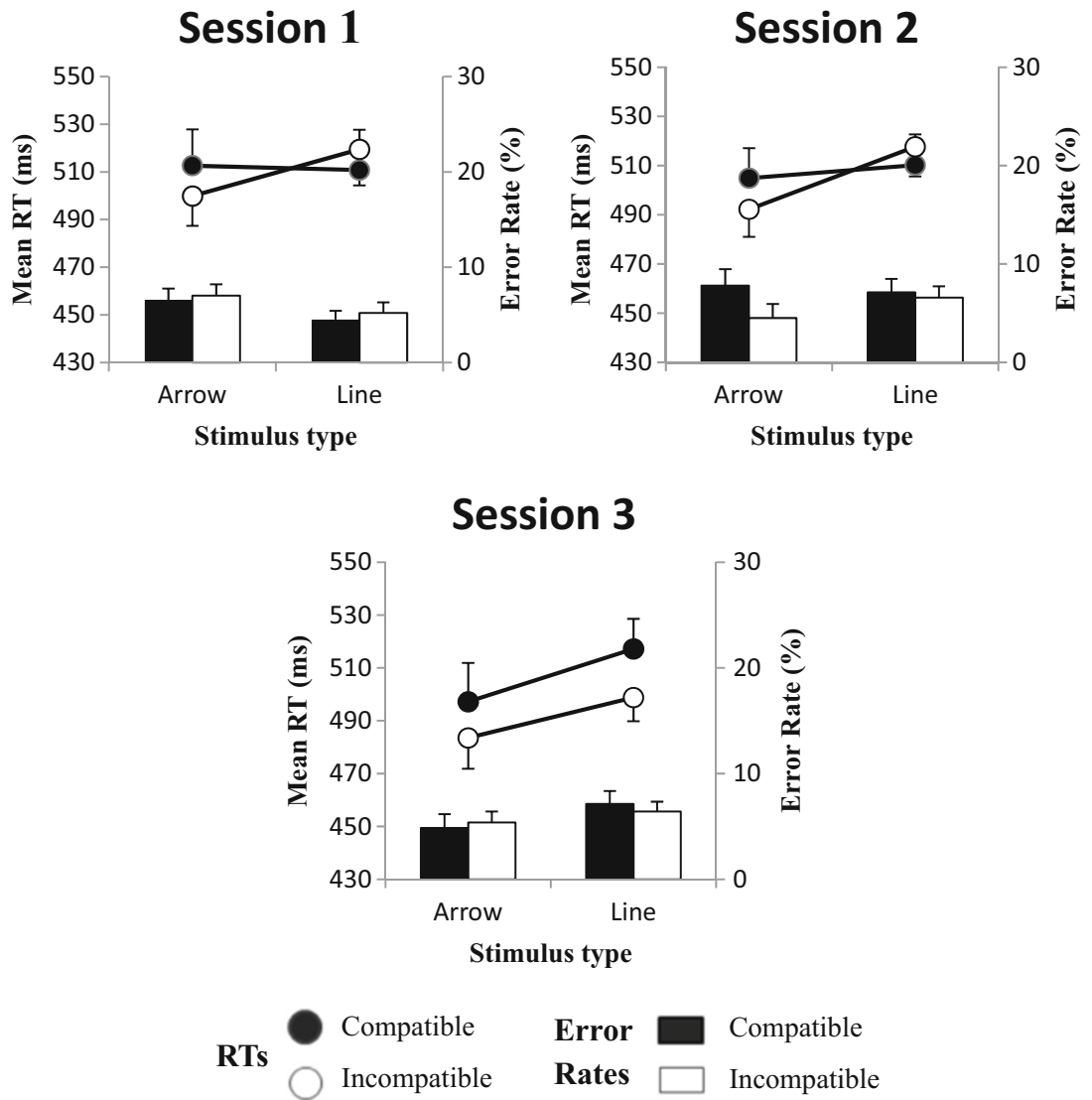

Fig. 2 Results of Experiment 1. Mean response times (RTs; line graphs) and mean error rates (bar graphs) in the condition of arrow and line stimuli for compatible and incompatible trials over three successive sessions in Experiment 1. Error bars show 1 standard error of the mean (SE)

MSE $=338.32]$, whereas significant main effect of compatibility $\left[\mathrm{F}(1,23)=8.68, p=.007, \mathrm{MSE}=713.49, \eta_{\mathrm{p}}{ }^{2}=.27\right]$ and marginally significant main effect of stimulus type $[\mathrm{F}(1,23)=$ $\left.3.68, p=.068, \mathrm{MSE}=2027.41, \eta_{\mathrm{p}}^{2}=.14\right]$ were observed. Paired $t$-tests confirmed significant NCEs in both the arrow $[t$ $(23)=3.21, p=.004, \mathrm{SE}=4.27]$ and line conditions $[t(23)=$ $2.21, p=.037, \mathrm{SE}=8.33]$. Additionally, in the arrow condition, the NCEs did not differ significantly among the three sessions [F $(2,69)=.02, p=.98]$, whereas in the line condition, the CEs differed significantly among the three sessions $[\mathrm{F}(2,69)=5.83, p=.005]$.

For the arcsine-transformed error rates, no significant main effect or interaction was observed (all $p \mathrm{~s}>.23$ ). However, paired $t$-tests confirmed a significant NCE in the arrow condition of Session $2[t(23)=2.84, p=.009, \mathrm{SE}=2.01]$, showing that the participants made fewer errors in incompatible trials $($ mean $=4.51 \%)$ than in compatible trials (mean $=$ $7.81 \%$ ). No other significant CE was observed.

There was no effect of any experimental manipulation on the arcsine-transformed Miss rates (all $\mathrm{Fs}<1.33$, all $p \mathrm{~s}>.31$ ). For the arcsine-transformed False Alarm rates, a significant difference was observed between stimulus types $[\mathrm{F}(1,23)=$ $\left.5.79, p=.025, \mathrm{MSE}=66.93, \eta_{\mathrm{p}}^{2}=.20\right]$, showing that the participants made fewer false alarms in line condition (mean $=10.88 \%)$ than in arrow condition (mean $=13.43 \%)$. No other significant difference was observed (all Fs $<1.87$, all $p$ s $>$ .17).

\section{Discussion}

The results from the FC task indicated that FC performance did not differ significantly from chance in either of the two stimulus conditions. This finding confirmed that primes were processed subliminally in the primary task.

In the primary task, NCEs appeared regardless of session and did not differ significantly among the sessions in the arrow stimuli condition. However, in the parallel-line stimuli condition, although no NCE appeared in Session 1 or 2, a significant NCE appeared in Session 3, and the CEs differed significantly among the three sessions. These results were consistent with the expectation that the automatization of associations plays a causal role in triggering motor activations. The findings indicated that stimuli with different properties can yield different strengths of S-R associations. Specifically, in the arrow stimuli condition, the strength of the $\mathrm{S}-\mathrm{R}$ associations was sufficiently strong that NCEs were observed. However, in 
the parallel-line stimuli condition, although the strength of the S-R associations was weaker in the early sessions such that no NCE was observed, when the strength of the S-R associations was reinforced by practice in Session 3, an NCE appeared. However, this experiment cannot completely exclude the role of response readiness in triggering motor activation, and the only way to learn anything about the role of response readiness is by comparing Experiments 1 and 2.

\section{Experiment 2}

\section{Methods}

\section{Participants}

Twenty-four paid college students (ten male) who ranged in age from 18 to 28 years (mean age $=21.58$ years) participated in the experiment. All of the participants were right-handed and had normal or corrected-to-normal vision. Informed consent was obtained from each participant at the start of the experimental session.

\section{Stimuli and apparatus}

The experimental stimuli and apparatus were similar to those described in Experiment 1.

\section{Procedure}

The experimental procedure was similar to the procedure described for Experiment 1, with the following differences: the No-Go targets were presented in $80 \%$ of all trials, and the Go targets were presented in $20 \%$ of all trials. One session consisted of four blocks (120 trials each) to achieve the same number of Go trials as in Experiment 1. In two blocks, parallel-line stimuli were used, and in the other two blocks, arrow stimuli were used. At the beginning of the experiment, the participants were informed that only $20 \%$ of the trials were Go trials.

\section{Results}

The data analysis of Experiment 2 was similar to the data analysis of Experiment 1. The performance of the FC task was assessed as follows: arrow stimuli condition, $48.75 \%$, and parallel-line stimuli condition, $49.44 \%$. Neither of the two conditions enabled the primes to significantly differ from chance $(50 \%)$, all were $t \mathrm{~s}(23)<1.09$ and $p \mathrm{~s}>.29$, which suggests that all primes were processed without awareness.

In the primary task, we also excluded outside of plus-minus three standard deviations from the RTs in each session for each participant (in total, $1.14 \%$ of the GO trials). Concerning the
RT, a significant difference was observed among the three sessions [F $(1.90,43.74)^{\mathrm{g}}=4.07, p=.026, \mathrm{MSE}=8412.05$, $\left.\eta_{\mathrm{p}}{ }^{2}=.15\right]$. The main effect of compatibility $[\mathrm{F}(1,23)=3.54, p$ $\left.=.073, \mathrm{MSE}=320.20, \eta_{\mathrm{p}}{ }^{2}=.13\right]$ or stimulus type $[\mathrm{F}(1,23)=$ $1.42, p=.25$, MSE $=5956.97]$ was not significant. The threeway interaction was not significant $\left[\mathrm{F}(1.71,39.41)^{\mathrm{g}}=.71, p=\right.$ $.48, \mathrm{MSE}=332.91]$. The two-way interaction between stimulus type and compatibility was significant $[\mathrm{F}(1,23)=13.54$, $\left.p=.001, \mathrm{MSE}=349.19, \eta_{\mathrm{p}}{ }^{2}=.37\right]$, and the two-way interaction between stimulus type and test session was also significant $\left[\mathrm{F}(1.90,43.67)^{\mathrm{g}}=3.42, p=.044, \mathrm{MSE}=3247.10, \eta_{\mathrm{p}}{ }^{2}=\right.$ .13]. The two-way interaction between test session and compatibility was not significant $\left[\mathrm{F}(1.78,40.82)^{\mathrm{g}}=.06, p=.93\right.$, MSE $=419.51]$. Additionally, we examined the interaction between stimulus type and compatibility regardless of session. Paired $t$-tests confirmed a significant NCE in the arrow condition $[t(71)=4.89, p<.001, \mathrm{SE}=2.47]$, showing that the mean RT for incompatible trials $($ mean $=553 \mathrm{~ms}$ ) was shorter than that for compatible trials (mean $=565 \mathrm{~ms}$ ). However, there was no significant $\mathrm{CE}$ in the line condition $[t(71)=$ $1.20, p=.24, \mathrm{SE}=3.45]$.

For the arcsine-transformed error rates, a significant difference was observed between stimulus types $[F(1,23)=12.96$, $\left.p=.002, \mathrm{MSE}=113.02, \eta_{\mathrm{p}}{ }^{2}=.36\right]$. However, the main effects of compatibility and test session were not significant (all $p s>$ .22). Additionally, the three-way interaction was not significant $\left[\mathrm{F}(1.62,37.25)^{\mathrm{g}}=3.05, p=.069, \mathrm{MSE}=83.23\right]$. The two-way interaction between test session and compatibility [F $\left.(1.53,35.11)^{\mathrm{g}}=.70, p=.47, \mathrm{MSE}=54.84\right]$ or between test session and stimulus type $\left[\mathrm{F}(1.87,43.00)^{\mathrm{g}}=.80, p=.45\right.$, MSE $=89.80]$ was not significant. The two-way interaction between compatibility and stimulus type was significant $[\mathrm{F}(1$, 23) $\left.=4.34, p=.048, \mathrm{MSE}=37.41, \eta_{\mathrm{p}}^{2}=.16\right]$. Next, we examined the interaction between stimulus type and compatibility regardless of session. However, paired $t$-tests confirmed no significant difference in either the arrow condition $[t(71)=$ $1.61, p=.11, \mathrm{SE}=1.18]$ or the line condition $[t(71)=.93, p=$ $.36, \mathrm{SE}=1.19]$. Figure 3 details the results of this experiment.

There was no effect of any experimental manipulation on the arcsine-transformed Miss rates (all Fs $<1.33$, all $p s>.31$ ). For the arcsine-transformed False Alarm rates, the interaction between test session and stimulus type was significant $[\mathrm{F}(1.58$, $\left.36.27)^{\mathrm{g}}=4.19, p=.031, \mathrm{MSE}=6.38, \eta_{\mathrm{p}}{ }^{2}=.15\right]$. Moreover, there is a significant difference between stimulus types of Session $2[t(23)=2.13, p=.044, \mathrm{SE}=.80]$, showing that the participants made fewer false alarms in line condition (mean = $0.82 \%$ ) than in arrow condition (mean $=1.32 \%$ ). No other significant difference was observed (all Fs $<1.20$, all $p s>.29$ ).

\section{Comparison of experiments 1 and 2}

Performance on the FC task, correct response RTs, arcsinetransformed error rates, and Miss rates on Go trials and 

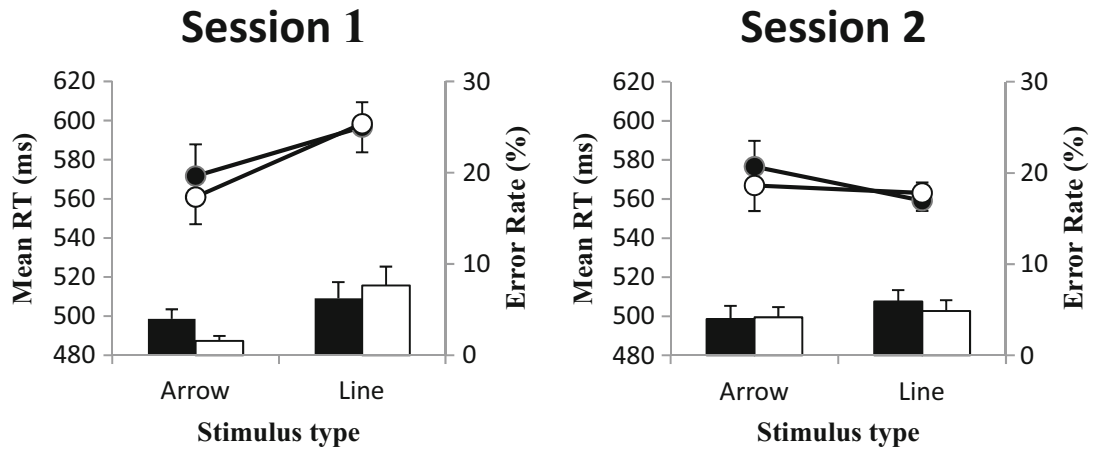

\section{Session 3}

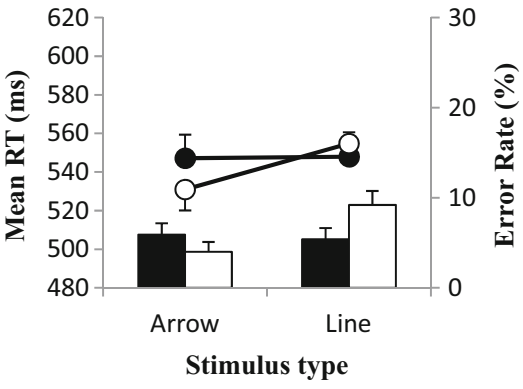

RTs

$\begin{array}{lll}\text { Compatible } & \text { Error } \square \text { Compatible } \\ \text { Incompatible } & \text { Rates } \square \text { Incompatible }\end{array}$

Fig. 3 Results of Experiment 2. Mean response times (RTs; line graphs) and mean error rates (bar graphs) in the condition of arrow and line stimuli for compatible and incompatible trials over three successive sessions in Experiment 2. Error bars show 1 standard error of the mean (SE)

arcsine-transformed False Alarm rates on No-Go trials were compared between Experiments 1 and 2. A mixed-design ANOVA was computed for performance on the FC task for the factors of stimulus type and experiment. Mixed-design ANOVAs were computed for the correct response RTs, the arcsine-transformed error rates and Miss rates on Go trials for the factors of test session (Sessions 1, 2, and 3), stimulus type (arrows, parallel lines), compatibility (compatible, incompatible), and experiment. In addition, a mixed-design ANOVA was computed for the arcsine-transformed False Alarm rates on No-Go trials for the factors of test session, stimulus type, and experiment.

No significant difference was observed in the performance of the FC task between Experiments 1 and $2[\mathrm{~F}(1,46)=.089$, $p=.77$ ], and no other significant effect was observed ( $p \mathrm{~s}>$ .29). The RTs were significantly shorter in Experiment 1 than in Experiment 2 (505 ms vs. $564 \mathrm{~ms}$; $\mathrm{F}(1,46)=47.71, p<$ $\left..001, \eta_{\mathrm{p}}{ }^{2}=.51\right)$. The main effects of compatibility $[\mathrm{F}(1,46)=$ $\left.10.23, p=.002, \mathrm{MSE}=403.40, \eta_{\mathrm{p}}{ }^{2}=.18\right]$ and test session $[\mathrm{F}$ $\left.(1.96,90.31)^{\mathrm{g}}=3.28, p=.043, \mathrm{MSE}=8679.08, \eta_{\mathrm{p}}{ }^{2}=.07\right]$ were significant. The two-way interaction between compatibility and stimulus type was significant $[\mathrm{F}(1,46)=26.53$, $p<.001$, MSE $\left.=272.86, \eta_{\mathrm{p}}{ }^{2}=.37\right]$. The CEs of Experiments 1 and 2 were compared in the arrow and line conditions. In the arrow condition, the NCEs did not differ significantly between Experiments 1 and $2[\mathrm{~F}(1,46)=.09$, $p=.77$ ], and no other significant effect was observed ( $p s>$ $.65)$. In the line condition, the CEs did not differ significantly between Experiments 1 and $2[\mathrm{~F}(1,46)=1.02, p=$ $.32]$, and the interaction between experiment and test session was significant $\left[\mathrm{F}(1.85,85.04)^{\mathrm{g}}=3.96, p=.026, \eta_{\mathrm{p}}^{2}\right.$ $=.08]$. Paired $t$-tests confirmed a significant difference in Session $3[t(23)=2.68, p=.013, \mathrm{SE}=9.45]$, showing that the NCE in Experiment 1 (-18 ms) was significantly larger than the $\mathrm{CE}$ in Experiment $2(+7 \mathrm{~ms})$, and no other significant difference was observed $(p s>.47)$. The error rates were not significant between Experiments 1 and 2 [F (1, $46)=2.02, p=.16]$. A significant main effect of stimulus type was observed $[\mathrm{F}(1,46)=12.10, p=.001$, MSE $=$ $\left.105.32, \eta_{\mathrm{p}}{ }^{2}=.21\right]$, showing that the error rate in the line condition (mean $=6.35 \%$ ) was higher than that in the arrow condition (mean $=4.75 \%$ ). There was no effect of any experimental manipulation on the arcsine-transformed Miss rates (all $\mathrm{Fs}<1.13$, all $p \mathrm{~s}>.32$ ). The arcsinetransformed False Alarm rate was significantly higher in Experiment 1 than in Experiment 2 (12.15\% vs. 1.01\%; F $\left.(1,46)=114.05, p<.001, \eta_{\mathrm{p}}{ }^{2}=.71\right)$ and was significantly higher in the arrow condition than in the line condition $(7.26 \%$ vs. $5.90 \% ; \mathrm{F}(1,46)=6.83, p=.012, \mathrm{MSE}=$ $\left.36.14, \eta_{\mathrm{p}}^{2}=.13\right)$. 


\section{Discussion}

The results from the FC task indicated that FC performance did not differ significantly from chance in either of the two stimulus conditions. This finding confirmed that primes were processed subliminally in the primary task. In the primary task, NCEs appeared regardless of session and did not differ significantly among the sessions in the arrow stimuli condition. However, no NCE appeared, regardless of session, and the CEs did not differ significantly among the sessions in the parallel-line stimuli condition.

Moreover, a comparison of Experiments 1 and 2 showed the following results. First, no significant difference was observed for discrimination performance in the FC task between the two experiments, which indicates that there was no significant difference in perceptual learning between the two experiments. Second, the RTs were significantly shorter in Experiment 1 than in Experiment 2, which indicates that response readiness can affect the execution of an overt response. Third, in the arrow stimuli condition, NCEs were observed regardless of session and experiment; moreover, there was no significant difference in the NCEs either among the sessions or between the experiments. However, in the parallelline stimuli condition, no significant $\mathrm{CE}$ appeared regardless of the experiment in Sessions 1 and 2, whereas a significant NCE was observed in Experiment 1 but not in Experiment 2 in Session 3. Moreover, the NCE in Experiment $1(-18 \mathrm{~ms})$ was significantly larger than the $\mathrm{CE}$ in Experiment $2(+7 \mathrm{~ms})$. Obviously, the results of Experiment 1 fully met the expectation of the automatization of associations, but the results of Experiment 2 did not. These results are consistent with the claim that the automatization of associations plays a causal role in triggering the motor activation for masked primes and that response readiness can modulate the development of automaticity. Specifically, the state of high response readiness (in Experiment 1) may have facilitated the development of automaticity such that the associations were automatized sufficiently in the parallel-line stimuli condition in Session 3, and NCE appeared. In contrast, in Experiment 2 the response readiness was not sufficiently high for the automatized associations to be effective, and no significant CE appeared. However, once the association is automatized, the modulation of response readiness will do little (i.e., in the arrow stimuli condition).

\section{General discussion}

\section{Overview of the study}

In the current study, two experiments were conducted to investigate the role of the automatization of associations and response readiness in triggering the motor activation for masked primes. If the automatization of associations played a causal role in triggering motor activations, we predicted that significant NCEs would emerge regardless of session and experiment in the arrow stimuli condition. In the parallel-line stimuli condition, we predicted that no significant NCE would appear early in practice; however, in subsequent sessions, NCEs would appear regardless of experiment. If response readiness was the only factor that played a causal role, we predicted that NCEs would appear regardless of stimulus type and session in Experiment 1. However, in Experiment 2, we predicted that no significant NCE would appear regardless of stimulus type and session. The results from the primary tasks revealed NCEs regardless of session and experiment in the arrow stimuli condition, and there was no significant difference for the NCEs either among sessions or between experiments. In the parallel-line stimuli condition, no significant CE appeared regardless of experiment in Sessions 1 and 2, whereas in Session 3, a significant NCE was observed in Experiment 1 but not in Experiment 2, and the NCE in Experiment 1 was significantly larger than the $\mathrm{CE}$ in Experiment 2. These results failed to completely satisfy the expectations of the automatization of associations or the response readiness because response readiness could modulate the development of automaticity. Specifically, high response readiness (i.e., Go 80\%) may have facilitated the development of automaticity such that the associations were automatized sufficiently in the parallel-line stimuli condition in Session 3, and an NCE appeared. In contrast, in Experiment 2, response readiness (i.e., Go 20\%) was not sufficient for effective automatized associations, and no significant $\mathrm{CE}$ appeared. Once the association is automatized (i.e., in the arrow stimuli condition), the modulation of response readiness will be diminished.

The modulation of response readiness to the development of automaticity may be attributed to the notion that low response readiness (e.g., induced by NO GO trials) can undo the automatization of associations; practice that can strengthen the associations (e.g., GO trials) can resist this undoing. In this study, when the number of GO trials is significantly larger than the number of NO-GO trials (in Experiment 1), the resistance to undoing will succeed, and the development of automaticity is facilitated. Thus, the associations were sufficiently automatized in the parallel-line stimuli condition in Session 3, and an NCE appeared. In contrast, when the number of NO$\mathrm{GO}$ trials is significantly larger than the number of GO trials (in Experiment 2), the resistance to undoing will fail, and the development of automaticity is not facilitated. Thus, the associations cannot be automatized, and no CE appeared.

The automatization of associations was not modulated by response readiness when the association was automatized (in the arrow stimuli condition). This result may be attributed to the notion that the resistance to undoing automatization is increased if the S-R relationship is highly compatible (e.g., 
arrow stimuli) such that the resistance will succeed when a response readiness is either high or low. Therefore, NCEs were observed regardless of session and experiment in the arrow stimuli condition.

It is possible that increasing the number of NOGO trials might directly influence the automatization of associations; in this case, response readiness may have no influence on the development of automaticity. However, previous research has indicated that response readiness can modulate motor activation at a sub-threshold level when the associations are weak. As mentioned in the introduction, previous studies have demonstrated that high response readiness, which is induced by experiment setting or instruction, such as a high level of alertness (Coles, Gratton, Bashore, Eriksen, \& Donchin, 1985) or fast response speed (Gratton, Coles, Sirevaag, Eriksen, \& Donchin, 1988), facilitates motor activation at a sub-threshold level when the associations are weak, and the authors deduced that response readiness is an energizing phenomenon through which response structures are activated at a sub-threshold level. Therefore, based on previous studies, the current study tended to favor the interpretation that response readiness modulates the development of automaticity.

\section{The role of the automatization of associations}

The current study indicates that the automatization of associations plays a causal role in triggering the motor activation for masked primes. Specifically, the NCE for masked primes was observed only when the association between specific stimuli and specific responses was automatized by prior responses to these stimuli. More specifically, this study's findings confirmed the role of self-automatization because the same set of stimuli was used for the primes and targets. In addition, Klapp and Greenberg (2009) confirmed that activation can occur only if the association has been automatized by auxiliary trials when different sets of stimuli are used for the primes and targets. In conclusion, the interpretation of the automatization of associations is supported.

\section{The role of response readiness}

In the current study, the RTs in Experiment 1 were significantly shorter than the RTs in Experiment 2, which is consistent with previous research (e.g., Miller, 1982, 1983; Schlaghecken \& Eimer, 2001). This result indicates that response readiness could affect the execution of an overt response. It may be because response inhibition, which is voluntary, optional and is closely related to executive mechanisms in the prefrontal cortex (Band \& van Boxtel, 1999), increases when response readiness is low. Moreover, the current study determined that response readiness could modulate the development of automaticity; thus, response readiness could indirectly affect subliminal priming. This finding may explain the need for response readiness to observe subliminal priming, which has been identified in some studies (Brunia, 1997; Coles, Gratton, Bashore, Eriksen, \& Donchin, 1985; Dosher \& Lu, 2000; Gratton, Coles, Sirevaag, Eriksen, \& Donchin, 1988; Rosenbaum, 1980; Welsh \& Elliott, 2004). These findings also indicate that top-down control can modulate the formation of association-based automaticity.

\section{Mechanism of the NCE}

The findings of the current study provide evidence for the SI hypothesis, which assumes that the strength of the prime activation determines the size of the CE. Weaker S-R associations can be strengthened through practice, and therefore, the motor activation of the prime would exceed the inhibitory threshold. Although no significant $\mathrm{CE}$ was observed in the early sessions, the NCE was observed in the subsequent session (in Experiment 1). This type of pattern could be attributed to an inhibition threshold in low-level motor control (Schlaghecken \& Eimer, 2000, 2002). Another possibility is that the results may be attributed to continuous subliminal inhibitory mechanisms (Liu et al., 2014), which assume that the onset of the inhibitory process in the subliminal inhibitory condition should delay (Lingnau \& Vorberg, 2005) and the inhibitory strength should decline (Houghton \& Tipper 1994) with decreasing activation of the prime. Then, the course of activation-to-inhibition may be similar for strong primes (e.g., arrow stimuli) and weak primes (e.g., neutral stimuli); however, the shift to inhibition sets should occur earlier for strong primes than for weak primes because arrow stimuli are strongly inhibited in the current study. Therefore, future research should attempt to explore the time-course of the shift to inhibition sets for stimuli of varying strength by prolonging the prime-target interval.

The MTI hypothesis assumes that an intervening stimulus (i.e., the mask) triggers inhibition and that the strength of this inhibition is relative to the features of the mask but not the prime. Specifically, a mask that contains relevant features should evoke strong inhibition, whereas an irrelevant mask should evoke weak inhibition (Jaśkowski, 2007). In the current study, the masks were irrelevant and invariable. According to the MTI hypothesis, the CEs should be constant in all conditions. The finding in the current study was not consistent with this expectation. However, the MTI hypothesis cannot be disregarded. Previous studies have demonstrated that an intervening stimulus (e.g., a distractor) triggers the inhibition of previously primed action even if this stimulus does not eliminate visibility of the prime.

\section{Perceptual learning}

Schlaghecken et al. (2008) reported that a prolonged FC task in which the masking stimulus remains constant across trials 
can cause perceptual learning that in turn can improve prime identification performance. This type of learning depends crucially on the constant masking stimulus because constancy enables participants to gradually learn to ignore the maski.e., to filter it out as irrelevant noise - and thus to perceive the relevant stimulus (Schubo, Schlaghecken, \& Meinecke, 2001). In the current study, although the masking stimulus remained constant across trials within each stimulus type condition, the results of the FC task confirmed that primes were processed subliminally in both Experiments 1 and 2. This result indicated that there is no perceptual learning in the experiments, possibly because the number of trials in the experiments was too low (lower than in Schlaghecken et al., 2008).

Although there were three times more No-Go trials in Experiment 2 than in Experiment 1, there was no significant difference in perceptual learning between these two experiments. There are two potential reasons for this result. First, the No-Go trials might not cause perceptual learning; i.e., although participants stared at a stimulating string throughout all trials, no perceptual learning occurred without overt response execution. Some studies have suggested that improved identification of simple stimuli involves two stages: neural fine-tuning that improved perceptual representations throughout the visual processing pathways in early visual areas (e.g., Sagi \& Tanne, 1994) in the former case, and filtering out the "perceptual noise" of the mask at subsequent, decision-related processing stages in the latter case (Dosher \& Lu, 1999; Maehara \& Goryo, 2003; Schubo et al., 2001). Schlaghecken et al. (2008) have argued that improved identification of backward pattern-masked stimuli is likely the result of late-stage learning instead of early learning at the level of the primary visual cortex. In the current study, however, backward pattern-masked stimuli were employed. Although the NoGo trials in Experiment 2 were three times more abundant than in Experiment 1, this type of No-Go trial involved no decision-related processing related to late-stage learning. Thus, the No-Go trials might not cause perceptual learning. Second, it is possible that a prolonged period of No-Go practice also leads to perceptual learning. It might be that there was no perceptual learning in this study because there were not enough No-Go trials. The lack of perceptual learning in this study, regardless of the reason, indicated that perceptual learning was controlled when investigating the role of the automatization of associations in triggering the motor activation for masked primes.

\section{Conclusion}

The automatization of S-R associations plays a causal role in triggering the motor activation for masked primes. Specifically, the NCE for masked primes is observed only if the association between specific stimuli and specific responses has been automatized by previous practice. Moreover, response readiness could modulate the development of automaticity, but this modulation effect may be diminished once the association is automatized. This finding shows for the first time that top-down control can modulate the formation of association-based automaticity.

Acknowledgments This study was supported by the National Natural Science Foundation of China under Grant Nos. 31371026 and 71601153, the Fundamental Research Funds for the Central Universities under Grant 2016CBY007, and the General Projects for Humanities and Social Science Research of Ministry of Education under Grant 16YJC190014.

\section{Compliance with ethical standards}

Funding This study was supported by the National Natural Science Foundation of China under Grants 31371026 and 71601153, the Fundamental Research Funds for the Central Universities under Grant 2016CBY007, and the General Projects for Humanities and Social Science Research of Ministry of Education under Grant 16YJC190014.

\section{References}

Band, G. P., \& van Boxtel, G. J. (1999). Inhibitory motor control in stop paradigms: Review and reinterpretation of neural mechanisms. Acta Psychologica, 101, 179-211. doi:10.1016/S0001-6918(99)00005-0

Bowman, H., Schlaghecken, F., \& Eimer, M. (2006). A neural network model of inhibitory processes in subliminal priming. Visual Cognition, 13(4), 401-480. doi:10.1080/13506280444000823

Boy, F., \& Sumner, P. (2010). Tight coupling between positive and reversed priming in the masked prime paradigm. Journal of Experimental Psychology. Human Perception and Performance, 36(4), 892-905. doi:10.1037/a0017173

Brunia, C. H. M. (1997). Gating in readiness. In P. J. Lang, R. F. Simons, \& M.-T. Balaban (Eds.), Attention and orienting: Sensory and motivational processes (pp. 281-306). Mahwah, NJ: Erlbaum.

Buckner, D. N., \& McGrath, J. J. (1963). Vigilance: A symposium. New York: McGraw-Hill Book Company.

Coles, M. G. H., Gratton, G., Bashore, T. R., Eriksen, C. M., \& Donchin, E. (1985). A psychophysiological investigation of the continuous flow model of human information processing. Journal of Experimental Psychology: Human Perception and Performance, 11, 529-533. doi:10.1037/0096-1523.11.5.529

Dosher, B. A., \& Lu, Z. L. (1999). Mechanisms of perceptual learning. Vision Research, 39, 3197-3221. doi:10.1016/S0042-6989 (99)00059-0

Dosher, B. A., \& Lu, Z. L. (2000). Mechanisms of perceptual attention in precuing of location. Vision Research, 40, 1269-1292. doi:10.1016/S0042-6989(00)00019-5

Eimer, M. (1999). Facilitatory and inhibitory effects of masked prime stimuli on motor activation and behavioural performance. Acta Psychologica, 101, 293-313. doi:10.1016/S0001-6918(99)00009-8

Eimer, M., \& Schlaghecken, F. (1998). Effects of masked stimuli on motor activation: Behavioral and electrophysiological evidence. Journal of Experimental Psychology: Human Perception and Performance, 24(1737-1747), 1737. doi:10.1037/0096-1523.24.6

Eimer, M., \& Schlaghecken, F. (2002). Links between conscious awareness and response inhibition: Evidence from masked priming. Psychonomic Bulletin \& Review, 9, 514-520. doi:10.3758/BF03196307 
Gibson, E. J. (1969). Principles of perceptual learning and development. New York: Appleton-Century-Crofts.

Gratton, G., Coles, M. G. H., Sirevaag, E. J., Eriksen, C. W., \& DoNchin, E. (1988). Pre- and post-stimulus activation of response channels: A psychophysiological analysis. Journal of Experimental Psychology: Human Perception and Performance, 14, 331-344. doi:10.1037 /0096-1523.14.3.331

Houghton, G., \& Tipper, S. P. (1994). A model of inhibitory mechanisms in selective attention. In D. Dagenbach \& T. H. Carr (Eds.), Inhibitory processes in attention, memory, and language (pp. 53112). San Diego: Academic Press, Inc.

Jaśkowski, P. (2007). The effect of nonmasking distractors on the priming of motor responses. Journal of Experimental Psychology: Human Perception and Performance, 33(2), 456-468. doi:10.1037/00961523.33.2.456

Jaśkowski, P. (2008). The negative compatibility effect with nonmasking flankers: A case for mask-triggered inhibition hypothesis. Consciousness and Cognition, 17(3), 765-777. doi:10.1016/j. concog.2007.12.002

Jaśkowski, P. (2009). Negative compatibility effect: The object updating hypothesis revisited. Experimental Brain Research, 193(1), 157160. doi:10.1007/s00221-008-1700-6

Jaśkowski, P., \& Slósarek, M. (2007). How important is a prime's gestalt for subliminal priming? Consciousness and Cognition, 16(2), 485497. doi:10.1016/j.concog.2006.06.005

Jaśkowski, P., \& Verleger, R. (2007). What determines the direction of subliminal priming. Advances in Cognitive Psychology, 3(1-2), 181-192. doi:10.2478/v10053-008-0024-1

Jaśkowski, P., Białuńska, A., Tomanek, M., \& Verleger, R. (2008). Maskand distractor-triggered inhibitory processes in the priming of motor responses: An EEG study. Psychophysiology, 45, 70-85. doi:10.1111/j.1469-8986.2007.00595.x

Klapp, S. T. (2005). Two versions of the negative compatibility effect: A reply to Lleras and Enns (2004). Journal of Experimental Psychology: General, 134, 431-435. doi:10.1037/0096-3445.134.3.431

Klapp, S. T. (2015). One version of direct response priming requires automatization of the relevant associations but not awareness of the prime. Consciousness and Cognition, 34, 163-175. doi:10.1016/j.concog.2014.08.004

Klapp, S. T., \& Greenberg, L. A. (2009). Temporary activation of perceptual-motor associations: A stimulus-response interpretation of automaticity. Journal of Experimental Psychology: Learning Memory \& Cognition, 35, 1266-1285. doi:10.1037 a 0016536

Klapp, S. T., \& Haas, B. W. (2005). Nonconscious influence of masked stimuli on response selection is limited to concrete stimulus Response as sociations. Journal of Experimental Psychology: Human Perception and Performance, 31(1), 193-209. doi:10.1037 /0096-1523.31.1.193

Klapp, S. T., \& Hinkley, L. B. (2002). The negative compatibility effect: Unconscious inhibition influences reaction time and response selection. Journal of Experimental Psychology: General, 131(2), 255269. doi:10.1037/0096-3445.131.2.255

Lingnau, A., \& Vorberg, D. (2005). The time course of response inhibition in masked priming. Perception \& Psychophysics, 67, 545-557. doi:10.3758/BF03193330

Liu, P., \& Wang, Y. (2014). Perceptual and motor contributions to the negative compatibility effect. Acta Psychologica, 153, 66-73. doi:10.1016/j.actpsy.2014.09.011

Liu, P., Chen, X., Dai, D., Wang, Y., \& Wang, Y. (2014). A subliminal inhibitory mechanism for the negative compatibility effect: A continuous versus threshold mechanism. Experimental Brain Research, 232(7), 2305-2315. doi:10.1007/s00221-014-3925-x

Low, K. A., \& Miller, J. (1999). The usefulness of partial information: Effects of go probability in the choice/nogo task. Psychophysiology, 36, 288-297. doi:10.1017/S0048577299980332
Maehara, G., \& Goryo, K. (2003). Perceptual learning in visual backward pattern masking. Perceptual and Motor Skills, 97, 1137-1149. doi:10.2466/pms.2003.97.3f.1137

Miller, J. (1982). Discrete versus continuous stage models of human information processing: In search of partial output. Journal of Experimental Psychology: Human Perception and Performance, 8, 273-296. doi:10.1037/0096-1523.8.2.273

Miller, J. (1983). Can response preparation begin before stimulus recognition finishes? Journal of Experimental Psychology: Human Perception and Performance, 9(2), 161-182. doi:10.1037/00961523.9.2.161

Neill, W. T., Terry, K. M., \& Valdes, L. A. (1994). Negative priming without probe selection. Psychonomic Bulletin \& Review, 1, 119121. doi:10.3758/BF03200767

Neumann, O. (1984). Automatic processing: A review of recent findings and a plea for an old theory. Cognition and motor processes, 255293. doi:10.1007/978-3-642-69382-3 17

Panis, S., \& Schmidt, T. (2016). What is shaping RT and accuracy distributions? Active and selective response inhibition causes the negative compatibility effect. Journal of Cognitive Neuroscience, 20, 121. doi:10.1162/jocn_a_00998

Posner, M. I. (1980). Orienting of attention. Quarterly Journal of Experimental Psychology, 32, 3-25. doi:10.1080/00335558008248231

Posner, M. I., \& Petersen, S. E. (1990). The attention system of the human brain. Annual Review of Neuroscience, 13, 25-42.

Rosenbaum, D. A. (1980). Human movement initiation: Specification of arm, direction, and extent. Journal of Experimental Psychology: General, 109, 444-474. doi:10.1037/0096-3445.109.4.444

Sagi, D., \& Tanne, D. (1994). Perceptual learning: Learning to see. Current Opinion in Neurobiology, 4, 195-199. doi:10.1016/09594388(94)90072-8

Schlaghecken, F., \& Eimer, M. (2000). A central-peripheral asymmetry in masked priming. Perception \& Psychophysics, 62, 1367-1382. doi:10.3758/BF03212139

Schlaghecken, F., \& Eimer, M. (2001). Partial response activation to masked primes is not dependent on response readiness. Perceptual and Motor Skills, 92, 208-222. doi:10.2466 /pms.2001.92.1.208

Schlaghecken, F., \& Eimer, M. (2002). Motor activation with and without inhibition: Evidence for a threshold mechanism in motor control. Perception \& Psychophysics, 64(1), 148-162. doi:10.3758 /BF03194564

Schlaghecken, F., \& Eimer, M. (2004). Masked prime stimuli can bias "free" choices be-tween response alternatives. Psychonomic Bulletin \& Review, 11(3), 463-468. doi:10.3758/BF03196596

Schlaghecken, F., \& Eimer, M. (2006). Active masks and active inhibition: A comment on Lleras and Enns (2004) and on Verleger, Jaśkowski, Aydemir, vander Lubbe, and Groen (2004). Journal of Experimental Psychology: General, 135(3), 484-494. doi:10.1037 /0096-3445.135.3.484

Schlaghecken, F., Blagrove, E., \& Maylor, E. A. (2007). Incidental learning of S-R contingencies in the masked prime task. Journal of Experimental Psychology: Human Perception and Performance, 33(5), 1177-1188. doi:10.1037/0096-1523.33.5.1177

Schlaghecken, F., Blagrove, E., \& May, E. A. (2008). No difference between conscious and nonconscious visuomotor control: Evidence from perceptual learning in masked priming task. Consciousness and Cognition, 17, 84-93. doi:10.1016/j. concog.2006.11.004

Schmidt, T., Hauch, V., \& Schmidt, F. (2015). Mask-triggered thrust reversal in the negative compatibility effect. Attention, Perception, \& Psychophysics, 77(7), 2377-2398. doi:10.3758/s13414-0150923-4

Schubo, A., Schlaghecken, F., \& Meinecke, C. (2001). Learning to ignore the mask in texture segmentation tasks. Journal of Experimental 
Psychology: Human Perception and Performance, 27, 919-931. doi:10.1037/0096-1523.27.4.919

Sumner, P. (2008). Mask - induced priming and the negative compatibility effect. Experimental Psychology, 55(2), 133-141. doi:10.1027 /1618-3169.55.2.133

Tipper, S. P., Weaver, B., Cameron, S., Brehaut, J. C., \& Bastedo, J. (1991). Inhibitory mechanisms of attention in identification and localization tasks: Time course and disruption. Journal of Experimental Psychology: Learning, Memory, \& Cognition, 17, 681-692. doi:10.1037/0278-7393.17.4.681

Welsh, T. N., \& Elliott, D. (2004). Effects of response priming and inhibition on movement planning and execution. Journal of Motor Behavior, 36, 200-211. doi:10.3200/JMBR.36.2.200211 